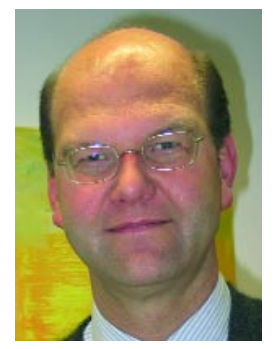

Martin Haupt

\title{
Psychotherapeutische und psychosoziale Maßnahmen
}

\author{
Martin Haupt, Schwerpunktpraxis: Hirnleistungsstörungen \\ Neuro-Centrum Düsseldorf
}

psychoneuro 2004; 30 (9): 475-480

$\mathrm{N}$ ichtmedikamentöse Optionen besitzen mittlerweile einen bedeutsamen Stellenwert im Gesamtbehandlungsplan der Demenz. Während sie sich noch vor 10-20 Jahren überwiegend auf unsystematische, kaum validierte und im weitesten Sinne supportive Maßnahmen für den Kranken und seine Bezugsperson beschränkten, stehen gegenwärtig gut strukturierte, validierte und zum Teil spezifische psychotherapeutische und psychosoziale Strategien zur Behandlung von Demenzkranken zur Verfügung (5).

Nach abgeschlossener Diagnostik sollte der behandelnde Arzt vor der Verordnung medikamentöser und nichtmedikamentöser Maßnahmen den Kranken und seine Familie gemäß seiner Informationspflicht adäquat über die Krankheit, ihren Verlauf und ihren Ausgang aufklären. Angesichts der Schwere des Leidens und der ungünstigen Prognose muss die Aufklärung dabei sehr behutsam erfolgen.

Die Therapie muss multimodal und integrativ sein und den individuellen Erfordernissen des Kranken und seiner Bezugspersonen gerecht werden. Alle bisher gemachten Erfahrungen mit therapeutischen Strategien, die sich nicht an diese Prämissen hielten, zeigten, dass die erzielten Effekte für den Kranken äußerst begrenzt waren.

Bei der irreversibel voranschreitenden Alzheimer Krankheit muss sich die Therapie gegenwärtig darauf beschränken, die Folgen der zerebralen Schädigung auf kognitive Leistung, Alltagsbewältigung und subjektives Wohlbefinden so weit und so lange wie möglich auszugleichen. Symptomatische Verbesserungen in

In den zurückliegenden 15 Jahren haben sich die nichtmedikamentösen Behandlungs- und Versorgungsmaßnahmen einen festen Platz im Gesamtbehandlungsplan bei Demenzerkrankungen gesichert. Zahlreiche Studien belegen mittlerweile den Nutzen für die Kranken und ihre Bezugspersonen. Bei den psychotherapeutischen und psychosozialen Maßnahmen sind es im wesentlichen stützende, beratende, psychoedukative sowie verhaltenstherapeutische Verfahren, die stadien- und settingabhängig eingesetzt werden. Nach wie vor können kognitive Trainingsbzw. Aktivierungsprogramme in der Verbesserung kognitiver Leistung bei überwiegend leichtgradig dementen Kranken nicht wirklich überzeugen. Studienberichte über deutliche Leistungsverbesserungen gehen in der Regel auf Untersuchungen mit zu kurzer Interventionsdauer zurück. Die Stärken der genannten Verfahren liegen aber eindeutig in der nachhaltigen Verbesserung des Wohlbefindens des Kranken, der Stabilisierung seines Affekts und Antriebs trotz fortschreitender Krankheit sowie in der stetigen Motivierung der Bezugspersonen zur Weiterführung der Pflege und in deren psychischer und physischer Entlastung im Pflegeprozess.

Teilleistungsbereichen, aber auch längerfristige Konsolidierungen des Funktionsniveaus sind bereits Indikatoren für einen Behandlungserfolg. Vorübergehende Verbesserungen von einzelnen Alltagseinbußen oder von Verhaltensauffälligkeiten sind in allen Stadien möglich. Für die praktische Behandlung im klinischen Alltag bedeutet dies, dass der behandelnde Arzt dem Kranken und seiner Familie zu jedem Zeitpunkt der Krankheit spürbare, wenn auch nur vorübergehende Verbesserungen der Krankheitsauswirkungen in Aussicht stellen kann.

Die mit der Demenz einhergehenden Veränderungen bestehen zum einen in kognitiven Leistungseinschränkungen und zum zweiten in krankheitsbedingten psychosozialen Beeinträchtigungen. Einen Überblick über die nicht-pharmakologischen Strategien gibt Tabelle 1.

\section{Kognitiv-aktivierende Interventionen}

Hiermit sind zunächst diejenigen kognitiven Verfahren gemeint, die mit neuropsychologischen Behandlungsmethoden eine direkte Verbesserung der kognitiven Leistungen und mittelbar auch der Gesamtbefindlichkeit des Kranken anstreben. Das kognitive Training eignet sich aufgrund seines Anforderungsprofils an den Kranken und seines methodischen Zugangs ausschließlich für leichtgradig demente Kranke und soll durch Steigerung der Gedächtnis- und Aufmerksamkeitsfunktionen die Selbständigkeit im Alltag in den ersten Jahren der Krankheit konsolidieren.

Bei Alzheimerkranken mit einer mittelgradig bis schwer ausgeprägten Demenz, die in Institutionen leben, wird bevorzugt das Realitäts-Orientierungs-Training (ROT) eingesetzt.

Dem Verlust der personalen Identität versucht die Selbst-Erhaltungs-Therapie entgegenzuwirken, die im wesentlichen auf den leichtgradig dementen Personenkreis abzielt. Ihr steht die Validations-Therapie nahe, deren Kernpunkt die Akzeptanz und das Verstehen der Welt des Alzheimerkranken ist.

\section{Wirksamkeit}

Frühere Untersuchungen zur Wirksamkeit von kognitiv zentrierten Therapieverfahren ergaben insge- 


\section{Tab. 1 Ausgewählte nicht-medikamen- töse Maßnahmen bei Demenzen}

Kognitiv-aktivierende Interventionen

- gegen einzelne kognitive Störungen (unimodal), gegen Störungsmuster (multimodal)

- Realitäts-Orientierungs-Training (ROT)

- Selbst-Erhaltungs-Therapie

- Validations-Therapie

Psychosoziale Maßnahmen

- Verhaltenstherapeutisch supportive Maßnahmen

- Psychoedukative Maßnahmen

- Beratung (rechtlich, finanziell, sozial)

samt keine überzeugenden Effekte (1-3). In einer vor wenigen Jahren durchgeführten Studie (6) wurde zur Leistungsverbesserung bei den Alzheimerkranken ein multimodales Training eingesetzt. Es bestand aus Übungen im Gebrauch von externalen Gedächtnishilfen, im Kategorisieren, Assoziieren und Visualisieren, Erlernen der zeitlichen und örtlichen Orientierung und aus dem Training des Wortschatzes und des Allgemeinwissens. Außerdem wurden psychotherapeutische und psychoedukative sowie motorische Therapieeinheiten verwendet. Die globale kognitive Leistungsfähigkeit blieb in der Trainingsgruppe im Unterschied zu einer leichten, nicht aber signifikanten Abnahme in der Kontrollgruppe konstant. Die Gruppe der Alzheimerkranken mit multimodalem Training war aber im Vergleich zur unbehandelten Gruppe deutlich weniger depressiv. Auch kam es, vermutlich als Ausdruck eines sozialen Gruppeneffektes, zu einem verbesserten zwischenmenschlichen Verhalten.

In einer eigenen Untersuchung führte die systematische Benutzung eines Notizbuches mit den wichtigsten persönlichen Aufzeichnungen und Hilfestellungen zu einer deutlich verbesserten Bewältigung von alläglichen Aufgaben. Dieser Effekt war auch für die Angehörigen erkennbar. Auch das Üben mit einem alltagsbezogenen interaktiven Computerprogramm kann die Alltagskompetenz verbessern (17). Vom kognitiven Training profitieren nach diesen Untersuchungen leichtgradig demente Kranken besonders dann, wenn die zu erlernende Information in engem Bezug mit der Alltagssituation des Kranken steht (17). Demgegenüber geht der erreichte Therapieeffekt kognitiver Interventionen bei leichtgradig dementen Kranken dann relativ rasch verloren oder stellt sich gar nicht erst ein, wenn innerhalb des Trainingsprogramms ausschließlich einzelne, nicht auf das individuelle Umfeld des Patienten konzentrierte kognitive Leistungen geübt werden.

In allen bisher durchgeführten Untersuchungen bei Demenzkranken konnte der Nachweis nicht erbracht werden, dass sich durch eine wie auch immer ausgeprägte Verbesserung einzelner kognitiver Funktionen das Gesamtfunktionsniveau des Kranken anheben ließ, bzw. dass sich der auf eine kognitive Funktion begrenzte Gewinn auf andere kognitive Leistungen nachhaltig transferieren ließ.

Kognitives Training eignet sich vermutlich auch für die mittlerweile zutreffender zu diagnostizierende Gruppe der Personen mit leichten kognitiven Störungen. Der wesentliche therapeutische Schwerpunkt dieser Interventionen in dem genannten Personenkreis, der sich im Vorfeld einer Demenz bewegt, müsste dabei in der Konsolidierung von relevantem Alltagswissen und in der verbesserten Fähigkeit, vorhandene Informationslücken auszufüllen, bestehen sowie in der Fertigkeit, schwerer abrufbare Informationen durch erlernte Umgehungsstrategien freizusetzen. Sofern auf diesem Wege für den Kranken spürbare Verbesserungen erreichbar wären, könnte dies auch mit einer präventiven Wirkung, bezogen auf die Entwicklung nichtkognitiver Störungen, etwa Depressionen, verbunden sein. Zukünftige Untersuchungen zur therapeutischen Wirksamkeit von nicht-medikamentösen Verfahren bei Personen mit leichten kognitiven Störungen sollten sich diesen Fragen verstärkt zuwenden.

\section{Realitäts-Orientierungs-Training}

Dieses, vor allem in Heimen und gerontopsychiatrischen Institutionen verbreitetes Trainingskonzept (9), setzt sich aus zwei Komponenten zusammen, dem „24 Stunden“-ROT oder auch informellem ROT sowie dem „classroom“-ROT oder auch formellem ROT. Das informelle ROT besteht in einer systematischen Gestaltung der Umgebung des Kranken mit dem wichtigsten Ziel, einen kontinuierlichen Fluss von orientierungsstiftenden Informationen und zudem ein adäquates Anforderungsniveau der Umgebung für den Kranken bereitzustellen. Die Mittel hierfür sind beispielsweise leicht erkennbare räumliche und zeitliche Orientierungspunkte, Hinweistafeln oder aktiv strukturgebendes Verhalten der Bezugspersonen. Das formelle ROT dagegen wiederholt in regelmäßigen Gruppensitzungen für den Alzheimerkranken die wichtigsten Informationen über den Alltag, insbesondere zu Zeit, Ort, Personen oder Tagesablauf. Das ROT führt unter Studienbedingungen zu Verbesserungen des Orientierungswissen (31), der sozialen Interaktion und der Kommunikation. Schließlich besteht ein günstiger Effekt auch in der behandlungsbedingt vermehrten Zuwendung der Bezugspersonen zum Kranken. Ein überdauernder Trainingseffekt war bisher aber nicht zu erkennen, was mit dem zumeist fortgeschrittenen Krankheitsschweregrad der beteiligten Demenzkranken zu erklären ist. Außerdem wurde eine Verbesserung des kognitiven Leistungsniveaus (27) sowie depressionsreduzierende, antriebsausgleichende und kognitiv stabilisierende Effekte berichtet $(10,20)$.

\section{Biographische Methoden}

Diese Methoden können im weitesten Sinne ebenfalls zu den kognitiven Interventionsverfahren gezählt werden. Ziel ist, die Erinnerung des Kranken an seine Lebensgeschichte möglichst aufrechtzuerhalten. Die Methode unterscheidet sich damit von den aus der Depressionsbehandlung bekannten Techniken, bei denen versucht wird, eine Aussöhnung mit der eigenen Biographie zu erzielen. Geeignete Materialien wie Bücher, Zeitungsausschnitte, Familienalben oder Musikstücke regen den Abruf von Erinnerungen an und erleichtern den Zugriff auf die eigenen Lebensdaten (23). Die dabei gewählten Interventionsmethoden sollten dem bestehenden Ausprä- 
gungsgrad der Krankheit angepasst sein. Die Durchführung kann in Einzelbehandlung oder in Gruppen sinnvoll sein. Sofern mit vom Demenzschweregrad homogenen Gruppen gearbeitet wird, ergeben sich häufig auch günstige Effekte auf die Kommunikation und das Zusammengehörigkeitsgefühl.

Selbst-Erhaltungs-Therapie (SET)

Die SET lässt sich als eine Kombination von Erinnerungstherapie und Validation einordnen (25). Durch Überlernen selbstbezogenen Wissens soll das Gefühl der Identität und der personalen Kontinuität erhalten werden. Dazu werden mit Hilfe der Familie vorbereitete gedächtnisstützende und reaktivierende Medien eingesetzt. Von Familien und Kranken wird diese Behandlungsform gut angenommen, weil sie sich auf einen relativ erhaltenen psychischen Funktionsbereich des Kranken bezieht, kaum den Kranken unmittelbar mit seinen Leistungsgrenzen konfrontiert, wie etwa das mnemotechnische Training, und die Familie aktiv einbezieht. Die Ergebnisse deuten darauf hin, dass sich im Verlauf dieser Therapie weniger die kognitiven als vielmehr die nichtkognitiven Symptome bessern. Im nichtkognitiven Symptombereich nehmen insbesondere depressive Auffälligkeiten ab (26).

\section{Validationstherapie}

Bei dieser Therapie wird die „Gültigkeit“ der Welt des Kranken in den Mittelpunkt der Betrachtung gerückt (7). Dies basiert auf der Hypothese, dass die übenden kognitiven und biographischen Verfahren zu sehr auf eine Verbesserung der Leistungsfähigkeit des Kranken gerichtet seien und ihn zu stark mit seinen durch die Krankheit gesetzten Grenzen konfrontierten. Die verwendeten Techniken bestehen in der nonverbalen Kommunikation sowie dem Einsatz von Musik und Lebenserinnerungen. Für die Bezugsperson ist es wichtig zu lernen, mit welchen Ausdrucksformen der Kranke auf sein Erleben und Empfinden verweist und wie er sich mit seinen Bedürfnissen im Alltag mitteilt. Be- stätigung und Sicherheit vermittelndes Verhalten sind wichtige Elemente. Validationstherapie wird insbesondere bei fortgeschrittenen Schweregraden der Demenzerkrankung eingesetzt. Die verbale Verständigung tritt dabei gegenüber der nonverbal geleiteten Kommunikation in den Hintergrund. Die Therapie führt $\mathrm{zu}$ einer Zunahme von Kommunikation und dem Bedürfnis nach sozialer Nähe (4). Sie wird mittlerweile in Weiterbildungen professionell Pflegender zunehmend vermittelt und in Pflegeinstitutionen eingesetzt. Wesentliches Ziel ist, die häufig in diesem Stadium der Demenz auftretenden Verhaltensstörungen von Dementen, die bei Konfrontation mit den eigenen Leistungsgrenzen entstehen, durch ein angemessenes, insbesondere Verständnis vermittelndes Verhalten der Bezugsperson abzufangen. Bisherige Ergebnisse zu dieser Methode reichen aber noch nicht aus, um ihre mögliche Wirksamkeit beurteilen zu können.

\section{- Psychosoziale Interventionen}

Diese Interventionsformen kann man unterteilen in verhaltenstherapeutische und psychoedukative Maßnahmen. Grundsätzlich steht im Rahmen der psychosozialen Behandlung die konstante und einfühlsame Beziehung zwischen Arzt und Patient bzw. seiner Familie im Mittelpunkt. Sie berücksichtigt zu jedem Zeitpunkt des Krankheitsverlaufs die enge Verschränkung von kognitiven und nichtkognitiven Symptomen und deren soziale Folgeerscheinungen.

Hilfestellungen bei der Wahrnehmung der noch vorhandenen Fähigkeiten und nicht nur Hilfe bei der Bewältigung der beeinträchtigten Leistungen sind im Umgang mit Demenzkranken äußerst bedeutsam. Depressiven Verstimmungen kann mit diesen therapeutischen Verhalten vorgebeugt werden. Aber auch wenn depressive Symptome bereits aufgetreten sind, insbesondere wenn sie reaktiv nach Überforderungssituationen oder einer Konfrontation mit den eigenen Leistungsgrenzen entstanden sind, sollte dem Kranken wieder Mut zugesprochen und Bestätigung vermittelt werden. Hierbei kann der Vermeidung von Tätigkeiten und dem Rückzug von sozialen Beziehungen und damit einer Verringerung von bestätigenden Erlebnissen entgegengewirkt werden. Dies trägt dazu bei, dass der Kranke sich trotz seiner zum Teil schmerzlich bewussten kognitiven Störungen bei seinen Bezugspersonen, etwa in der Familie, aufgehoben weiß und Unterstützung und Zuspruch erfährt. Bei länger andauernden oder schwerer ausgeprägten depressiven Aufälligkeiten sind solche Strategien besonders wichtig und bedürfen eines strukturierten Einsatzes durch professionelle Therapeuten.

\section{Wirksamkeit}

Die Wirksamkeit konnte in einer kontrollierten randomisierten Untersuchung bei 72 leichtgradig dementen depressiven Kranken eindrucksvoll bestätigt werden (28). Eine katamnestische Erhebung sechs Monate nach Abschluss der dreimonatigen Behandlung zeigte, dass die Demenzkranken noch immer bessere Werte im Sinne einer geringeren Depression aufwiesen im Vergleich zu ihren Werten beim Eingang in den Behandlungszeitraum. Die Ergebnisse dieser Studie konnten darüber hinaus belegen, dass auch die Angehörigen von der Intervention bei ihren $\mathrm{zu}$ betreuenden demenzkranken Partnern profitierten. Demnach ist eine in die Beziehung des Kranken mit seiner Bezugsperson gerichtete psychotherapeutische Intervention für beide Partner der Beziehung positiv wirksam, unabhängig davon, welcher der beiden Partner im Mittelpunkt der Intervention steht. Wird der Demenzkranke erfolgreich behandelt, kann sich auch das Befinden der Bezugsperson bessern und umgekehrt. Der Umstand, dass ein derartiger „Brückeneffekt“ bei psychotherapeutischen Interventionen in diesem Setting beobachtet werden kann, zeigt bereits für ein leichtes Stadium der Demenz sehr deutlich die eng verflochtenen Beziehungsstrukturen zwischen den Kranken und ihren Bezugspersonen. Das therapeutische Potential, das in einer derartigen Intervention wirksam werden kann, übersteigt daher auch 
bei weitem den Effekt, der allein an der Person zu verzeichnen ist, auf die die Intervention unmittelbar gerichtet war.

\section{Bei psychotischen Störungen}

Bei Wahn und Halluzinationen oder Verkennungen der Umgebung bzw. von Personen können vorbeugende Strategien kaum entwickelt werden. Zunächst können Ablenkungsmaßnahmen versucht werden, aber auch eine klare Strukturierung der Umgebung und eine Überschaubarkeit des Alltags sind wichtig. Mitunter lassen sich Fehleinschätzungen des Kranken als Folge von Situationsverkennungen aber auch im voraus vermeiden, z.B. durch das Entfernen des Spiegels, wenn das Spiegelbild zum feindlichen Aggressor wird, oder durch das Ausschalten des Fernsehers, wenn der gezeigte Kriminalfilm Anlass zu Verfolgungsängsten gibt, oder auch durch das Zuziehen des Vorhangs am Abend, wenn das Zimmerlicht die Person des Kranken vor dunklem Hintergrund im Fenster spiegelt und der Kranke sich fälschlicherweise von außen dadurch bedroht oder beobachtet glaubt.

\section{Bei motorischer Unruhe und Weglauftendenzen}

Milieutherapeutische Interventionen sind verhaltenstherapeutische Strategien, die dem Kranken in jedem Krankheitsstadium durch eine gezielte Anpassung der Lebensbedingungen eine optimierte Anpassung an seine Umgebung ermöglichen sollen. Infolge einer derartigen Modifizierung der Umgebung soll sich der Grad der Ablesbarkeit („Dekodierung“) für den Demenzkranken erhöhen und damit seine Selbständigkeit erhalten bleiben (16). So ist beispielsweise bekannt, dass motorisch unruhige, unablässig sich fortbewegende Demenzkranke in Pflegeheimen meist einem von der Umgebung gegebenen Impuls folgen, etwa einer an der Wand gezeichneten Farblinie oder einem eingerichteten Handlauf. Erzwungene Richtungswechsel, etwa an Flurenden, bereiten ihnen Schwierigkeiten, da sie vermutlich Gefühle willkürlich gesetzter Begrenzung hervorrufen, die Ängstlichkeit bewirken kön- nen, aber auch Ärger oder aggressives Verhalten. Zur Vermeidung solcher für den Kranken und seine Umgebung nachteiligen Reaktionen auf potentiell aversiv wirkende Umgebungsbedingungen tragen zum Beispiel gemütlich gestaltete Sitzgelegenheiten am Flurende bei oder Flurgänge, die als Endloswege angelegt sind, damit Ausgangs- und Endpunkt des Weges identisch werden (14). Durch Aufstellung eines mannshohen Spiegels am Stationsausgang (22) sowie durch das Aufkleben eines zweidimensional wirkenden Gittermusters am Boden (18) konnte die Häufigkeit der Kontakte mit der Ausgangstür, als Hinweis auf ein drohendes Verlassen der Station, jeweils um rund ein Drittel reduziert werden.

\section{Snoezelen bei Antriebsstörungen} und Impulskontrollverlust

Bei Kranken in fortgeschrittenen Krankheitsstadien bieten sich Maßnahmen zur sensorischen Stimulation durch haptisch anregende Materialien und angenehme Gerüche an. Die Nutzung von „Snoezelen“-Räumen in einigen Pflegeeinrichtungen hat sich zur Beruhigung von Antriebsstörungen und übermäßigem Impulskontrollverlust bei schwerer beeinträchtigten Demenzkranken in der Alltagspraxis gut bewährt (16).

\section{Bei Agitiertheit und aggressivem Verhalten}

Für die gezielte Intervention gegen Agitiertheit und aggressives Verhalten bei leicht- bis mittelgradig beeinträchtigten Dementen können verhaltenstherapeutische Maßnahmen erfolgreich sein. Die Verhaltensstörungen können mit einer standardisierten, manualgeleiteten, verhaltenstherapeutischen Intervention deutlich reduziert werden (29). Kernpunkte der multimodalen Intervention waren die individuell zugeschnittene Kombination aus bewegungstherapeutischen, tagesstrukturierenden und angenehme Erlebnisse bereitstellenden Elementen für die Demenzkranken sowie die Vermittlung von Problemlösestrategien bei schwer bewältigbaren Situationen mit den Kranken und von Strategien zum Selbstmanagement für die betreuenden Bezugspersonen.

Die Wirksamkeit wurde in weiteren Fallbeispielen untermauert (11). Auch bei diesen Formen der therapeutischen Intervention, die sich im wesentlichen an den Kranken richtete, war ein positiver „Brückeneffekt“ für die Bezugsperson zu verzeichnen. Nach abgeschlossener Intervention gaben sie an, schwierige Situationen mit dem Demenzkranken selbstständig besser als zuvor bewältigen zu können.

Einbeziehung der Angehörigen

Eine für Demenzkranke und ihre Bezugspersonen geeignete psychosoziale Behandlung, die in der häuslichen Umgebung stattfand, stellten Hinchliffe und Mitarbeiter vor (15). Dabei wurden in einem kontrollierten Design den 33 teilnehmenden Familien über vier Monate mit einem individuell zugeschnittenen Behandlungsplan gezielte psychotherapeutische Maßnahmen, eine unter Umständen erforderliche psychopharmakologische Behandlung und eine soziale Beratung zur Verfügung gestellt. Am Ende der Intervention war bei den behandelten Kranken eine um 75\% gegenüber dem Behandlungsbeginn verminderte Häufigkeit von Verhaltensstörungen nachweisbar; die Häufigkeitsabnahme von Verhaltensproblemen betrug bei den unbehandelten Kranken hingegen nur 25\% (15). Für die betreuenden Angehörigen, auf die sich ebenfalls die therapeutische Intervention richtete, war, gemessen mit dem General Health Questionnaire, ebenfalls ein positiver therapeutischer Nutzen zu verzeichnen.

\section{Gruppentherapie und Paarinterventionen}

Auch eine systemisch orientierte, familientherapeutische Aspekte einbeziehende Gruppenintervention mit pflegenden Angehörigen von Demenzkranken (Tab. 2) vermindert den Belastungsgrad durch die Pflege (siehe Beitrag von Stoppe \& Geilfuss in diesem Heft). Die Motivation zur Fortsetzung der häuslichen Pflege kann so deutlich angehoben werden. In informierten und beratenen Familien wird eine erheblich geringere Rate an Heimunter- 
bringungen erreicht. Angehörigenarbeit ist auch in Bezug auf die Häufigkeit und Intensität von nichtkognitiven Störungen der Demenzkranken therapeutisch wirksam (12).

Paartherapeutische Interventionen sind ebenfalls erfolgreich (13), wie eine offene, nichtkontrollierte Studie mit 32 Paaren (überwiegend mittelschwer beeinträchtigte Demenzkranke und pflegende Angehöriger) zeigte. Diese Studie basierte erstmals auf einer unmittelbaren Kooperation der örtlichen Universitätsklinik für Psychiatrie und Psychotherapie mit der regionalen Alzheimer Gesellschaft. Schwerpunkte der Gruppenarbeit waren Themen aus dem individuellen sozialen Umfeld, den erforderlichen hauswirtschaftlichen Aufgaben, dem vorhergegangenen Berufs- und Freizeitleben. Die Angehörigen wurden intensiv mit den eingeschränkten, aber auch erhaltenen Fertigkeiten und Fähigkeiten der Kranken vertraut gemacht. Kontinuierlich wurden problematische Interaktionen in der Pflegebeziehung aufgegriffen und mit den Angehörigen Lösungsmöglichkeiten gemeinsam erarbeitet. Insgesamt handelte es sich bei dieser Zusammenstellung von fördernden, interaktiven Aufgaben um eine multimodale Intervention zur Stärkung und Konsolidierung qualitativ unterschiedlicher Gestaltungs- und Wahrnehmungsleistungen des Gehirns (13). Die Ergebnisse zeigen im wesentlichen, dass sich bei den Kranken das adäquate Kommunikationsverhalten mit den pflegenden Partnern signifikant verstärkte. Bei den pflegenden Angehörigen wurden die Verhaltensmuster im Umgang mit den Kranken signifikant verbessert und eine spürbare Entlastung trotz fortgesetzter Pflege herbeigeführt. Diese Untersuchungsergebnisse weisen darauf hin, dass gezielte psychoedukative und paartherapeutisch orientierte Interventionen bei Demenz über den Zeitraum von nahezu einem halben Jahr zu einer Konsolidierung des Funktionsniveaus des Kranken und einer Verbesserung seiner Kommunikation führen, sowie zu einer bedeutsamen Entlastung der pflegenden Angehörigen und Steigerung ihrer Pflegemotivation (13). Dies war unabhängig von der verabreichten Medikation mit Antidementiva oder von der Gabe von psychopharmakologischen Substanzen (13).

In einer weiteren kürzlich vorgelegten Studie von Marriott und Mitarbeitern (21) wurde der Gruppenarbeit mit pflegenden Anghörigen eine auf die Erfordernisse der Alzheimer Krankheit zugeschnittene Version der kognitiv-behavioralen Familienintervention bei schizophren Kranken zugrunde gelegt. Wie auch in anderen Untersuchungen zur Wirksamkeit von Gruppenarbeit mit pflegenden Angehörigen zeigte sich allein in der Interventionsgruppe eine bedeutsame Verbesserung des Befindens der Angehörigen. Es wurde eine deutliche Reduktion von subjektiver Belastung und Depressivität festgestellt. Bei den Demenzkranken kam es, im Sinne des „Brückeneffektes“, ebenfalls zu Verbesserungen, die sich vor allem im Bereich der zu Studienbeginn erhobenen Verhaltensstörungen nachweisen ließen (21). Die Demenzkranken der Kontrollgruppen besserten sich in keinem der erhobenen kognitiven und nichtkognitiven Parameter. Auch für die pflegenden Angehörigen der Kontrollgruppen waren keine bedeutsamen therapeutischen Effekte nachweisbar. Die dargelegten und diskutierten Ergebnisse der aufgeführten Untersuchungen zur Gruppenarbeit mit pflegenden Angehörigen haben, unabhängig von ihrem jeweiligen methodischen Zugang eindrucksvoll gezeigt, dass sowohl die Angehörigen als auch die Angehörigen profi- tierten. Gruppenarbeit mit pflegenden Angehörigen von Demenzkranken ist damit ein unverzichtbarer Bestandteil in der Betreuung von Familien mit Demenzkranken (30). Um so mehr auch deswegen, weil pflegende Angehörige von Demenzkranken infolge der langwierigen Beanspruchung durch die Versorgung des Kranken eine Hochrisikogruppe für psychische und somatische Erkrankungen und für die erhöhte und unkontrollierte Einnahme von beruhigenden Medikamenten sind.

\section{Bei fortgeschrittener Demenz}

Neuere Übersichtsarbeiten weisen darauf hin, dass psychosoziale Interventionen auch in fortgeschrittenen Stadien der Demenz für den Kranken wirksam sein können (8). Selbst bei schwerst beeinträchtigten Alzheimerkranken sind über basale Aktivitäten stimulierende, musikoder bewegungstherapeutische Interventionen spürbare Verbesserungen im Bereich der Stimmung und des Verhaltens zu erzielen. Aktivierende, regelmäßig vorgenommene Massagemaßnahmen und Akupressur mit hautverträglichen Ölen können beispielsweise eine spürbare Wirkung auf Unruhezustände, aggressives Verhalten, aber auch gezielte Aufmerksamkeitsleistungen von schwer dementen Kranken erzielen (19). Eine ähnlich positiver Effekt wird auch bei sensorischer Stimulation beispielsweise dem „Snoezelen“ beobachtet (5).

\section{- Ausblick}

Ein gravierender Nachteil der gegenwärtig verfügbaren Datenlage besteht darin, dass die Untersuchungen zur Wirksamkeit von psychoso-

\section{Tab. 2 Themenbereiche in einem verhaltenstherapeutisch orientierten psychoedukativen Gruppenprogramm mit pflegenden Angehörigen von Demenzkranken (12)}

- Symptome, Verlauf, Diagnose, Behandlung von Demenzen

- Umgang mit der konkreten Pflegesituation

- Besondere Rolle der nicht-kognitiven Symptome

- Beziehung zwischen pflegenden Angehörigen und Kranken

- Spezifische emotionale Probleme der Angehörigen

- Unterstützung der Pflege durch das soziale Umfeld

- Umgang mit juristischen und finanziellen Problemen 
zialen Interventionen, bezogen auf die verwendeten Stichproben, das methodische Vorgehen oder die Dauer der Behandlung, teilweise erheblich voneinander abweichen (24). Auch wurden bisher noch keine Studien vorgelegt, in denen bestimmte pharmakologische und nichtpharmakologische Maßnahmen kombiniert oder differentiell gegenübergestellt wurden. So wäre beispielsweise für den Zeitpunkt der Heimunterbringung zu hoffen, dass sich die deutlich prolongierenden Effekte der Azetylcholinesterase-Hemmer und die der psychoedukativen Angehörigenarbeit zum Nutzen des Kranken und seiner Familie miteinander verbinden ließen. Bereits heute ist aber ausreichend belegt, dass im Gesamtbehandlungsplan der Demenz die kognitiven und psychosozialen nicht-pharmakologischen Interventionen ein notwendiger Bestandteil der medizinischen und psychologischen Versorgung sind.

\section{Psychotherapeutic and psycho- educative interventions}

Within the last 15 years nonpharmacological interventions in dementia have become an unequivocal part of the therapeutic concept. A considerable amount of literature confirms the beneficial effects of these interventions for the dementia sufferers as well as for their caregivers. Among psychotherapeutic and psychosocial strategies competent support, counseling, psychoeducative interventions and behavioural therapy play the most important role. Programmes exclusively activating cognitive abilities are still not convincing with regard to improvement of dementia core symptoms. The therapeutic strength of these interventions presents with a long-term improvement of the well-being of patients, a consolidation of affect and behaviour in spite of the progressive disease and in a sustaining motivation of families to continue with caring combined with a reduction of stress and strain in everyday life.

\section{Key words:}

dementia - psychotherapeutic/ psychoeducative interventions treatment effects

\section{Literatur}

1. Beck C, Heacock P, Mercer S. The impact of cognitive skills remediation training on persons with Alzheimer's disease and mixed dementia. J Geriatr Psychiatr 1988; 21: 73-88

2. Bourgeois MS. Enhancing conversation skills in patients with Alzheimer's disease by using a prosthetic memory aid. J Appl Beh Analysis 1990; 23: 29-42

3. Camp CJ, Schaller JR. Epilogue: spacedretrieval memory training in an adult daycare center. Educ Gerontol 1989; 15: 641-648

4. Cheston R. Psychotherapeutic work with people with dementia: a review of the literature. $\mathrm{Br}$ J Med Psychol 1998; 71 : 211-231

5. Cohen-Mansfield J. Nonpharmacologic interventions for inappropriate behaviors in dementia. Am J Geriatr Psychiatr 2001; 9 : 361-381

6. Ermini-Fünfschilling D, Meier D. Gedächtnistraining: wichtiger Bestandteil der Milieutherapie bei seniler Demenz. Z Gerontol Geriatr 1995; 28: 190-194

7. Feil N. Validation. Ein neuer Weg zum Verständnis alter Menschen. Wien: Delle Karth, 1990

8. Finnema $E$ et al. The effects of emotionoriented approaches in the care for persons suffering from dementia: a review of the literature. Int J Geriatr Psychiatr 2000; 15: 141-161

9. Folsom JC. Reality orientation for the elderly mental patient. J Geriatr Psychiatr 1968; 1:291-307

10. Gaugler JE et al. Respite for dementia caregivers: the effects of adult care service use on caregiving hours and care demands. Int Psychogeriatr 2003; 15: 37-58

11. Haupt M. Kognitiv-behavioristische Interventionen bei Aggressivität im Alter. In: Hirsch R, Radebold M (Hrsg.). Verhaltenstherapie bei Älteren. Bonn: Tagungsband der 11. Jahrestagung für Psychotherapie im Alter, 2000

12. Haupt $M$ et al. Verbesserung von Unruhezuständen und Angst bei Demenzkranken nach psychoedukativer Gruppenarbeit mit pflegenden Angehörigen. Fortschr Neurol Psychiatr 2000; 68: 216-223

13. Haupt $M$ et al. Behandlungseffekte einer paartherapeutischen psychoedukativen Gruppenarbeit mit Demenzkranken und ihren pflegenden Angehörigen. Fortschr Neurol Psychiatr 2000b; 68: 503-515

14. Heeg S. Bauliches Milieu und Demenz. In: Wahl HW, Tesch-Römer C (Hrsg.). Angewandte Gerontologie in Schlüsselbegriffen. Kohlhammer, 2000: 233-241

15. Hinchliffe AC et al. Behavioural complications of dementia. Int J Geriatr Psychiatr 1995; 8: 111-113

16. Höwler E. Gerontopsychiatrische Pflege - Lehr- und Arbeitsbuch für die Altenpflege. Hagen: Kunz, 2000

17. Hofmann M, Berner C, Müller-Spahn F. Interaktives Computertraining bei Alzheimer-Patienten. Nervenheilkunde 2001; 2: 104-107

18. Hussian RA, Brown DC. Use of two-dimensional Grid patterns to limit hazardous ambulation in demented patients. J Gerontol 1987; 42: 558-560

19. Kilstoff K, Chenoweth L. New approaches to health and well-being for dementia day-care clients, family carers and day care staff. Int J Nurs Pract 1998; 4: 70-83

20. Lai CKY, Chi I, Kayser-Jones J. A randomised controlled trial of a specific reminiscence approach to promote the well-being of nursing-home residents with dementia. Int Psychogeriatr 2004; 16:33-49

21. Marriott A et al. Effectiveness of cognitive-behavioural family intervention in reducing the burden or care in carers of patients with Alzheimer's disease. $\mathrm{Br}$ J Psychiatr 2000; 176: 557-562

22. Mayer R, Darby SJ. Does a mirror deter wandering in demented older people? Int J Geriatr Psychiatr 1991; 6: 607-609

23. Norberg A. Caring for demented patients. Acta Scand Neurol Suppl 1996; 165 : 105-108

24. Opie J, Rosewarne R, O'Connor DW. The efficacy of psychosocial approaches to behaviour disorders in dementia: a systematic literature review. Aust N Z J Psychiatr 1999; 33: 789-799

25. Romero B. Selbst-Erhaltungs-Therapie (SET): Betreuungsprinzipien, psychotherapeutische Interventionen und Bewahren des Selbstwissens bei Alzheimerkranken. In: Weis S, Weber G (Hrsg.). Handbuch Morbus Alzheimer. Weinheim, Psychologie Verlags Union, 1997; 1209-1221

26. Romero B, Wenz M. Konzept und Wirksamkeit eines Behandlungsprogrammes für Demenzkranke und deren Angehörige. Z Gerontol Geriatr 2002; 35:118-128

27. Spector A et al. Efficacy of an evidencebased cognitive stimulation therapy programme for people with dementia. Br J Psychiatr 2003; 183: 248-254

28. Teri $L$ et al. Behavioral treatment of depression in demented patients: a controlled clinical trial. J Gerontol 1997; 4: 159-166

29. Teri L, Logdson RG, Weiner MF, Trimmer C, Thal L, Whall AL, Peskind E. Treatment for agitation in dementia patients: a behavior management approach. Psychotherapy 1998; 35: 436-443

30. Teri L. Training families to provide care: effects on people with dementia. Int J Geriatr Psychiatr 1999; 14: 110-119

31. Zanetti $O$ et al. Reality orientation therapy in Alzheimer's disease: useful or not? A controlled study. Alz Dis Ass Disord 1995; 9: 132-138

\section{Korrespondenzadresse:}

PD Dr. Martin Haupt

Schwerpunktpraxis Hirnleistungsstörungen Neuro-Centrum (Neurologie, Psychiatrie, Psychotherapie, Neuroorthopädie) Düsseldorf

Hohenzollernstraße 1-5

40211 Düsseldorf 